\title{
EVALUATION OF OPTICAL COHERENCE TOMOGRAPHY PATTERNS IN DIABETIC MACULAR OEDEMA
}

\author{
Venkitasubramanian Mallika ${ }^{1}$,Vaikkakara Sudha² \\ 1 Assistant Professor, Department of Ophthalmology, Government Medical College, Thrissur. \\ ${ }^{2}$ Associate Professor, Department of Ophthalmology, Government Medical College, Thrissur.
}

\section{ABSTRACT}

\section{BACKGROUND}

The aim of this study is to identify, categorise and analyse the Optical Coherence Tomography patterns of Diabetic Macular oedema.

\section{MATERIALS AND METHODS}

In this observational study, 43 eyes of 25 patients with Diabetic Macular oedema (DME) were evaluated. DME was defined as the retinal thickening due to fluid leakage and pooling in the macular area in patients with Diabetes Mellitus. Macular oedema due to other ocular illness was excluded. All patients underwent best corrected visual acuity assessment by Snellen's visual acuity chart, dilated slit-lamp Biomicroscopic examination, Fundus Fluorescein Angiography(FFA) and Optical Coherence Tomography (OCT) by the same examiner. OCT patterns were analysed. Central foveal thickness was also measured by OCT and macular oedema classified into mild $(201 \mu \mathrm{m}-300 \mu \mathrm{m}$,) moderate $(301 \mu \mathrm{m}-400 \mu \mathrm{m})$ and severe $(\geq 400 \mu \mathrm{m})$.

\section{RESULTS}

Of the total 25 patients in the age group 35-75 years (Mean age 54.08), males predominated in this study (M: F ratio of 2.6:1). OCT examination revealed that $30 \%$ eyes had Cystoid macular oedema and $26 \%$ had Sponge-like retinal thickness. Mixed cystoid and spongiform pattern $\mathrm{w}$

as observed in 28\%, Epiretinal membrane (ERM) in 9\%, Plaque of hard exudates in 7\%, Serous macular detachment in $9 \%$, and Vitreomacular traction in 5\%. 32\% eyes had mild macular oedema, 21\% had moderate and 35\% had severe forms.

\section{CONCLUSION}

Various patterns can be easily identified by OCT and treatment may be modified accordingly. Cystoid macular oedema was the predominant form of DME according to this study. Both eyes of a same patient can present with different DME patterns.

\section{KEYWORDS}

Optical Coherence Tomography, Diabetic Macular oedema, Cystoid Macular Oedema, Epiretinal Membrane, Central Foveal Thickness, Vitreomacular Traction.

HOW TO CITE THIS ARTICLE: Mallika V, Sudha V. Evaluation of optical coherence tomography patterns in diabetic macular oedema. J. Evolution Med. Dent. Sci. 2017;6(20):1571-1574, DOI: 10.14260/Jemds/2017/345

\section{BACKGROUND}

Diabetic Macular Oedema (DME), a microvascular complication which is caused by the breakdown of the bloodretinal barrier, promotes neuroglial dysfunction and concomitant visual disturbance. ${ }^{1}$ It is the commonest cause of visual loss in patients with non-proliferative diabetic retinopathy and a common cause of visual loss in proliferative diabetic retinopathy.

Diabetic macular oedema is diagnosed stereoscopically as retinal thickening in the macula using slit-lamp biomicroscopy. The ETDRS defined DME as retinal thickening or presence of hard exudates within 1 DD of the centre of the macula. To characterise the severity of macular oedema, and for treatment guidelines the term Clinically Significant Macular Oedema (CSME) is used. Macular oedema is clinically

Financial or Other, Competing Interest: None.

Submission 15-01-2017, Peer Review 22-02-2017,

Acceptance 02-03-2017, Published 09-03-2017.

Corresponding Author:

Vaikkakara Sudha,

Associate Professor,

Department of Ophthalmology,

Government Medical College, Thrissur.

E-mail: ajisudha@gmail.com

DOI: $10.14260 /$ jemds $/ 2017 / 345$ significant, if one of the following conditions is present: 1 . Retinal thickening at or within $500 \mu$ of the centre of the macula. 2. Hard exudates at or within $500 \mu$ of the centre of the macula if associated with thickening of retina. 3 . A zone or zones of retinal thickening one disc area or larger, any part of which is within one disc diameter of the centre of the macula. ${ }^{2}$

Diabetic macular oedema tends to be a chronic disease. Although spontaneous recovery is not uncommon, $24 \%$ of eyes with CSME and $33 \%$ of eyes with centre involving CSME will have a moderate visual loss (15 or more letters on the ETDRS chart) within 3 years if untreated. ${ }^{3}$

The incidence of DME over a 10 -year period was $20.1 \%$ among patients diagnosed before age 30 years (younger onset) and $39.3 \%$ among patients diagnosed after 30 years. ${ }^{4}$ As the severity of overall retinopathy increases, the proportion of eyes with macular oedema also increases. 3\% in eyes with mild non-proliferative diabetic retinopathy (NPDR), 38\% with moderate-severe NPDR and 71\% with proliferative diabetic retinopathy (PDR) develop DME. 5

Optical coherence tomography (OCT) is a fast and noninvasive tool for examining the retina in cross sectional images that correlate reasonably with the retinal histology. It is not only helpful in detecting DME early, but has the added advantage of being able to reveal not only the presence of cystoid macular oedema, but subfoveal serous retinal 
detachment, vitreomacular traction or an epiretinal membrane which cannot be detected in FFA.

Moreover, the macular thickness map gives us a very accurate idea of central retinal thickness and can quantify the degree of improvement or worsening following therapy.

\section{Aim of Study}

The aim of the study was to identify, categorise, and analyse the OCT patterns of Diabetic Macular Oedema.

\section{MATERIALS AND METHODS}

This was an observational study done between October 2010 and March 2011 in patients who attended the retina clinic of Govt. Medical College, Thrissur. 43 eyes of 25 patients with Diabetic Macular Oedema were evaluated. The study group included both insulin dependent and non-insulin dependent proliferative diabetic retinopathy and non-proliferative diabetic retinopathy between the ages of 35-75 years.

None of the patients in our study had undergone previous focal laser or pan-retinal photocoagulation, or ocular surgery in the past six months. Other exclusion criteria were dense cataract, macular oedema owing to other ocular illness and advanced diabetic retinopathy.

Diabetic macular oedema is diagnosed stereoscopically as retinal thickening in the macula. The patients were diagnosed as having Diabetic Macular Oedema by slit-lamp biomicroscopic examination with 90D lens. A detailed history regarding onset of visual loss and duration of diabetes, treatment taken, etc was taken and recorded. All these patients underwent best corrected visual acuity assessment by Snellen's visual acuity chart, and dilated slit-lamp biomicroscopic examination. Fundus photographs were taken and macular oedema was confirmed by Fundus Fluorescein Angiography. Spectral domain OCT (OPKO) was taken on the same day, by the same examiner. OCT was done in all eyes, a line scan program was chosen and the image processed and analysed for pattern characterisation. Central macular thickness was measured with the retinal thickness map. Macular oedema was categorised into mild (with a thickness of $201-300 \mu)$, moderate $(301-400 \mu)$ and severe $(\geq 400 \mu)$.

\section{RESULTS}

Of the 25 patients we analysed, there were $3(12 \%)$ patients in the age group 30-39 years, 2 (8\%) in 40-49 years age group, 11 (44\%) in 50-59 years age group, 8 (32\%) in 60-69 age groups and $1(4 \%)$ in $70-79$ age group. Males predominated with M: F ratio of 2.6:1. 67.3\% had NPDR and $32.7 \%$ PDR. Mean diabetic age was 14.08 years.

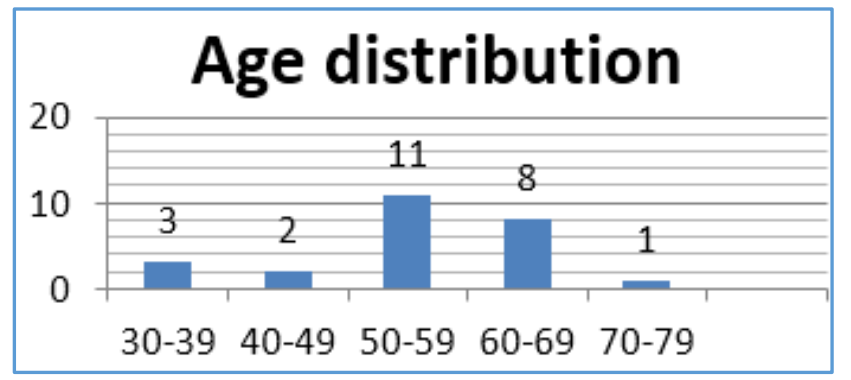

Figure 1. Age Distribution of the Patients in the Study

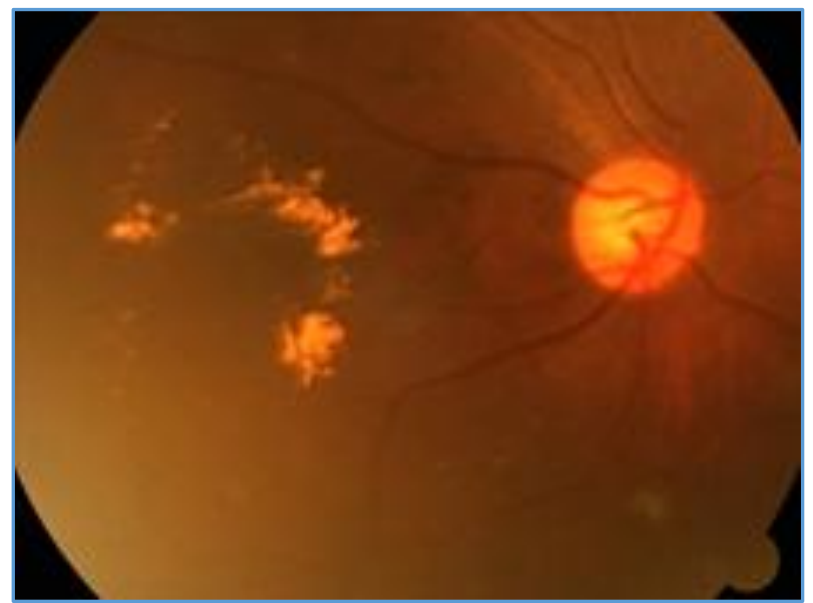

Figure 2. Fundus Picture of DME

Biomicroscopic examination of all the patients showed Diabetic macular oedema. $11 \%$ patients showed DME associated with cystoid macular oedema (CME), and 2\% had DME with vitreomacular traction (VMT). No patients had Epiretinal membrane (ERM) or Serous Macular Detachment with Subretinal Fluid (SRF) clinically.

Ocular Coherence Tomography analysis showed seven patterns of macular oedema in our patients.

\section{Spongiform Pattern}

Eyes with spongy oedema showed diffuse thickening of macula. It mostly involved the outer retinal layers, while the internal layers maintained their normal reflectivity. Cross sectional scans show swelling of the retina giving it a spongy appearance with increased retinal thickness.

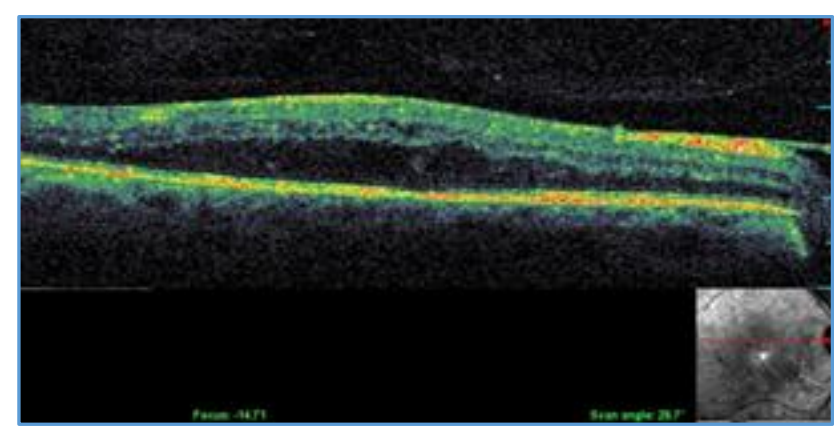

Figure 3. Spongiform Oedema on OCT

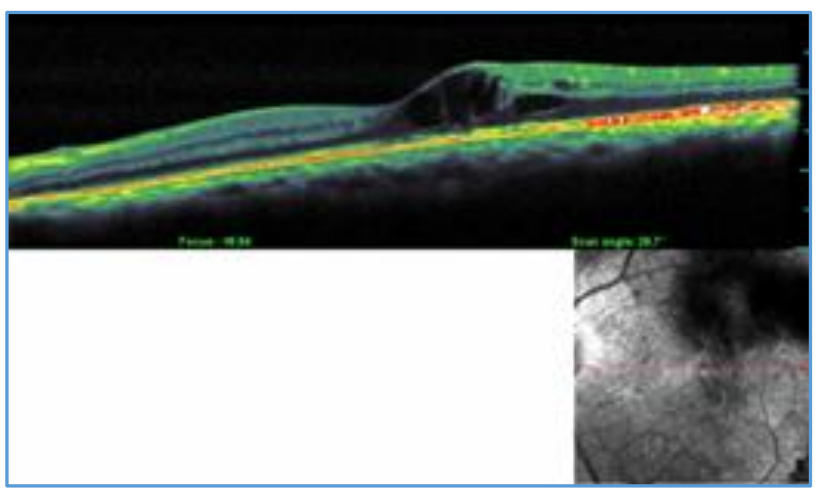

Figure 4. CME on OCT 


\section{Cystoid Pattern}

Eyes with CME showed large cystic spaces in the foveolar and parafoveal region. It involves various depth of retina and has intervening septa in between.

\section{Mixed Pattern}

Some eyes showed both spongiform thickening of outer retinal layers and cystoid spaces in the inner retina.

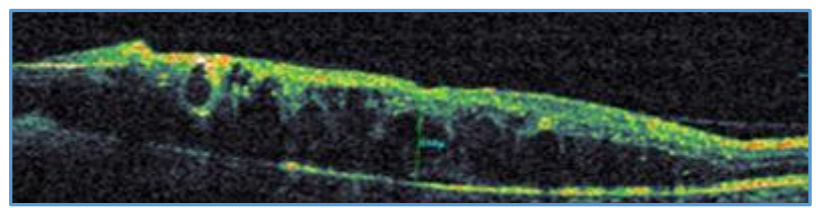

Figure 5. Mixed Spongiform and Cystoid Oedema on OCT

Serous Macular Detachment was seen as a hyporeflective area between neurosensory retina and RPE.

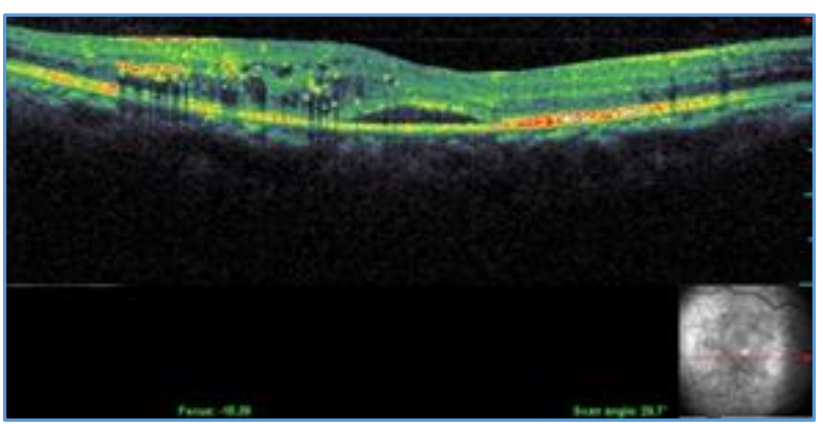

Figure 6. Serous Subfoveal Detachment

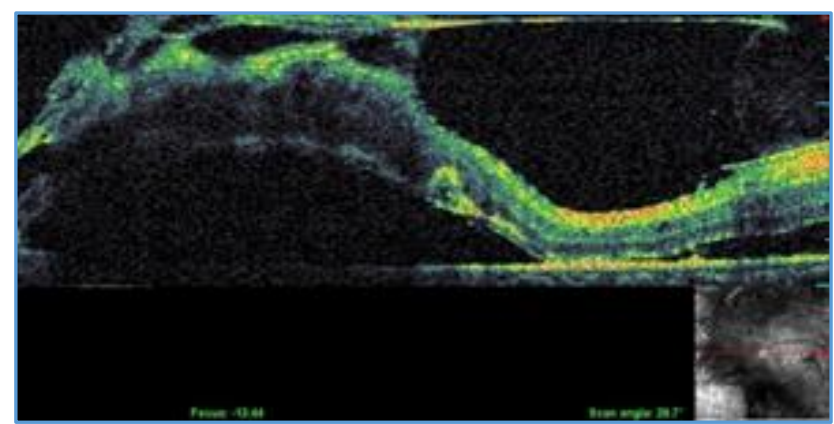

Figure 7. VMT

Vitreomacular Traction or VMT was seen as hyperreflective band in the vitreous, which was adherent to the fovea, either centrally or paracentrally causing traction and pulling up the macula.

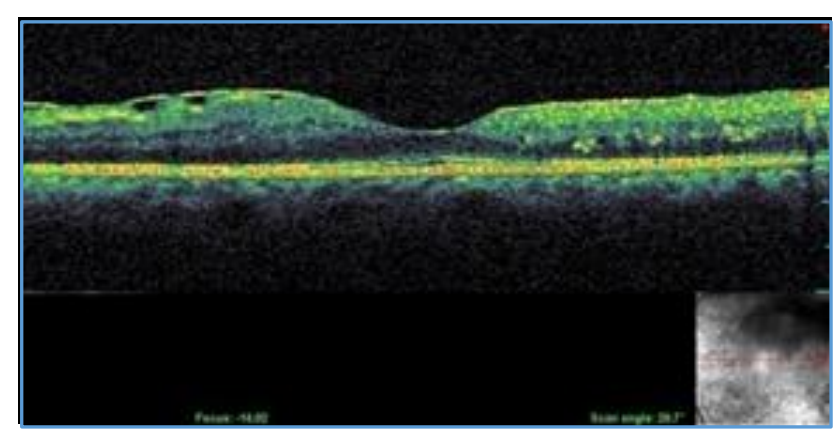

Figure 8. ERM Figure

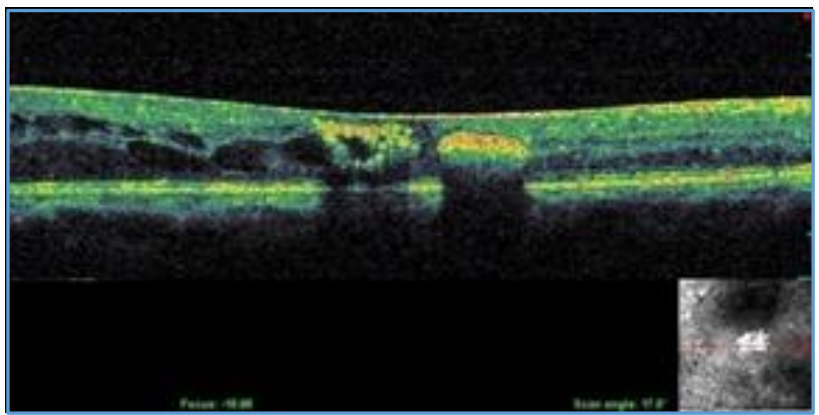

Figure 9. Hard Exudates Plaques on OCT

Epiretinal membrane or ERM was identified as a hyperreflective thickening at the level of ILM, causing distortion and flattening of the foveal surface.

Hard Exudates Plaque was seen as hyper-reflective intraretinal plaque which cast a shadow due to the blockade of light transmission.

\begin{tabular}{|c|c|}
\hline Pattern of DME in OCT & \% of Eyes \\
\hline Spongy oedema & 26 \\
\hline Cystoid oedema & 30 \\
\hline $\begin{array}{c}\text { Mixed spongy and cystoid } \\
\text { oedema }\end{array}$ & 28 \\
\hline ERM & 9 \\
\hline Submacular detachment & 9 \\
\hline VMT & 5 \\
\hline Hard exudate plaques & 7 \\
\hline Table 1. Distribution of Eyes with Various OCT Patterns in \\
Patients with DME \\
\hline
\end{tabular}

$15 \%$ of patients presented with differing OCT patterns in both eyes.

\begin{tabular}{|c|c|c|}
\hline Type of DME & Biomicroscopy & OCT \\
\hline CME & $11 \%$ & $30 \%$ \\
\hline SRF & $\mathrm{Nil}$ & $9 \%$ \\
\hline VMT & $2 \%$ & $5 \%$ \\
\hline ERM & $\mathrm{Nil}$ & $9 \%$ \\
\hline \multicolumn{2}{|c|}{ Table 2. Comparison of DME Characteristics Identified by } \\
\end{tabular}

Measurement of macular thickness revealed 33\% of eyes with mild macular oedema, 21\% moderate oedema and 35\% with severe diabetic macular oedema.

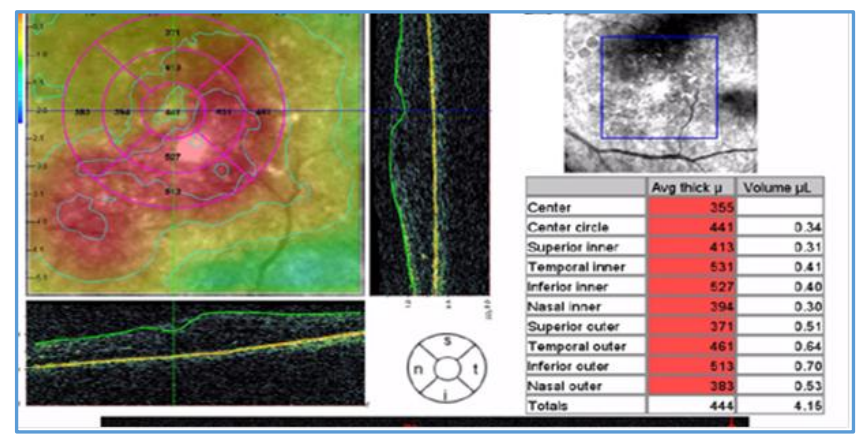

Figure 10. Macular Thickness Mapping 


\section{DISCUSSION}

Optical Coherence Tomography is a fast and noninvasive tool for examining the retina in cross sectional images that correlates reasonably with the retinal histology. Till recently slit-lamp biomicroscopy and FFA were the tools for the diagnosis and management of DME. It is true that these are highly sensitive for the qualitative detection of DME. OCT enables us to detect and understand the accurate subclinical retinal changes associated with DME that may not be detectable even in FFA. Yang et al have suggested that OCT may be more sensitive than clinical examination in assessing DME and is a better tool for documenting changes in macular thickening. In his series, OCT identified spongy retinal thickness seen in $58 \%$ of eyes. ${ }^{6}$ Otani et al found spongy retinal thickness in $88 \%$, CME in $47 \%$, SRF in $15 \%$ of eyes with CSME. Kim et al found spongy retinal swelling in $97 \%$, CME in $55 \%$, SRF in $7 \%$, VMT in $13 \%$ of eyes with DME. 7 Ozdek et $\mathrm{al}^{8}$ had reported spongy swelling in 66\%, CME in $16 \%$, SRF in $10 \%$ of eyes with DME. In our series, cystoid macular oedema was the common form of presentation. Our study revealed that $26 \%$ had macular thickening with spongy oedema, $30 \%$ with cystoid changes, $28 \%$ with mixture of spongy and cystoids oedema, 9\% ERM, 9\% with serous retinal detachment, 5\% with vitreomacular traction and $12 \%$ with plaques of hard exudates. The higher incidence of cystoid form of macular oedema in our series could be due the fact that the section of diabetic population presenting to our retina clinic is with longer diabetic age and thus their diabetic macular oedema a longstanding one. CME pattern represents a chronologically later stage of DME.

In our study, $30 \%$ of the eyes had CME on OCT, compared to $11 \%$ detected by biomicroscopy. Ozdek et al also found that $40 \%$ of CME detected on OCT were not detected by biomicroscopy and 63\% were not detected even by FFA. Thus, OCT tends to be a better diagnostic tool in detecting CME than biomicroscopy or FFA.

In our study, 9\% of eyes had SRF with subfoveal retinal detachment, which could not be detected by biomicroscopy. Most series have found SRF in 8-12\% of eyes with DME.

According to our study, 5\% had VMT as per OCT and 2\% as per biomicroscopy. VMT has been reported by various authors between $10-60 \%$ of eyes with DME.

Another important finding of our study was both eyes of a same patient can present with different DME patterns.

\section{CONCLUSION}

Diabetic macular oedema is a major cause of visual disability in diabetic patients. DME may be more easily and accurately diagnosed in an early stage with OCT as compared to slitlamp biomicroscopic examination. Various patterns can be easily identified and treatment may be modified accordingly. Being noninvasive, its acceptance as a followup imaging modality to monitor the course of DME and response to therapy is high. It helps to selectively identify cases like VMT and ERM which needs surgical intervention.

\section{REFERENCES}

[1] Murakami T, Nishijima K, Sakamoto A, et al. Foveal cystoid spaces are associated with enlarged foveal avascular zone and microaneurysms in diabetic macular edema. Ophthalmology 2011;118(2):359-67.

[2] Early Treatment Diabetic Retinopathy Study Research group. Treatment techniques and clinical guidelines for photocoagulation of diabetic macular edema. ETDRS Report2. Ophthalmology 1987;94(7):761-74.

[3] Bhagat N, Grigorian RA, Tutela A, et al. Diabetic macular edema: pathogenesis and treatment. Surv Ophthalmol 2009;54(1):1-32.

[4] Anderson JM, Van Itallie CM. Tight junctions and the molecular basis for regulation of paracellular permeability. Am J Physiol 1995;269(4 Pt 1):G467-75.

[5] Klein R, Klein BE, Moss SE. Visual impairment in diabetes. Ophthalmology 1984;91(1):1-9.

[6] Yang CS, Cheng CY, Lee FL, et al. Quantitative assessment of retinal thickness in diabetics patients with and without CSME using OCT. Acta Ophthalmol Scand 2001;79(3):266-70.

[7] Otani T, Kishi S, Maruyama Y. Patterns of diabetic macular edema with OCT. Am J Ophthalmol 1999;127(6):688-93.

[8] Ozdek SC, Erdinc MA, Guerlik G, et al. Optical coherence tomographic assessment of diabetic macular edema: comparison with fluorescein angiographic and clinical findings. Ophthalmologica 2005;219(2):86-92. 